\title{
Are cystic features natural course of the late phase in COVID-19 pneumonia?
}

Noriko HIRAI $^{1}$, Ryotaro Kida ${ }^{1}$, and Hiraku Yanada ${ }^{1}$

${ }^{1}$ Asahikawa Medical University

July 18, 2021

\begin{abstract}
A patient with late in COVID-19 pneumonia presented peripheral cystic features on chest computed tomography, which were spontaneously resolved with no antibiotic therapy or surgery. Physicians should pay attention to follow up the late phase of COVID-19 pneumonia for better understanding its etiologies and clinical course in the convalescing period.
\end{abstract}

CLINICAL IMAGE

Are cystic features natural course of the late phase in COVID-19 pneumonia?

Noriko Hirai * (correspondence)

Respiratory Center, Asahikawa Medical University

Address: 2-1-1 Midorigaoka-Higashi, Asahikawa, Hokkaido, Japan

Phone: +81-166-69-3290

Fax: +81-166-69-3299

Email: a020034@asahikawa-med.ac.jp

Ryotaro Kida

Respiratory Center, Asahikawa Medical University

Address: 2-1-1 Midorigaoka-Higashi, Asahikawa, Hokkaido, Japan

Phone: +81-166-69-3290

Fax: +81-166-69-3299

Email: rkida@asahikawa-med.ac.jp

\section{Hiraku Yanada}

Respiratory Center, Asahikawa Medical University

Address: 2-1-1 Midorigaoka-Higashi, Asahikawa, Hokkaido, Japan

Phone: +81-166-69-3290

Fax: +81-166-69-3299

Email: yana12@asahikawa-med.ac.jp

Keywords : COVID-19, pneumonia, cyst, pneumothorax, computed tomography 


\section{Key clinical message}

Cystic features are thought as a part of natural course of COVID-19 pneumonia caused by ischemia or fibrosis in late phase. Clinicians should pay attention to follow up the late phase of COVID-19 pneumonia for better understanding its etiologies.

\section{Abstract}

A patient with late in COVID-19 pneumonia presented peripheral cystic features on chest computed tomography, which were spontaneously resolved with no antibiotic therapy or surgery. Physicians should pay attention to follow up the late phase of COVID-19 pneumonia for better understanding its etiologies and clinical course in the convalescing period.

\section{CLINICAL IMAGE}

A 35-year-old man with fever, cough, and worsening short of breath was hospitalized with COVID-19 pneumonia. His chest computed tomography $(\mathrm{CT})$ showed bilateral patchy consolidation and ground-glass opacities (GGO) (Figure 1A). He received oxygen and ciclesonide inhalation. Mechanical ventilation was not required. Twenty-six days after, CT on discharge showed a cystic feature in the left mediastinal side (Figure 1B). As he was physically recovered and the blood test did not show any inflammatory findings, only CT follow-up was conducted. Further four weeks later, a new cystic feature enclosed by thickened wall were found in the epiphrenic side (Figure 1C). Again he was asymptomatic with no abnormality in the blood test. Further four weeks later, both cysts had spontaneously disappeared without intervention (Figure 1D).

COVID-19 pneumonia is a viral infection which is reported to present with GGO, patchy shadowing, and interstitial abnormalities. ${ }^{1}$ Cystic features and pleural involvement are less reported except for mechanical ventilation.$^{1,2}$ Its etiology is still poorly understood, but it may be caused by parenchymal ischemic damage and fibrosis. ${ }^{2}$ We want to emphasize that physicians should pay attention to follow up the late phase of COVID-19 pneumonia for better understanding its clinical course in the convalescing period.

Conflict of interest disclosure statement: None declared.

Ethical Approval : Not required.

Patient Consent : The patient provided written informed consent for his data to be published.

Author contributions : NH designed the conception, acquisition of the data and all authors wrote the manuscript.

Data availability statement : Data sharing is not applicable to this article as no new data were created or analyzed in this study.

\section{References}

1. Guan WJ, Ni ZY, Hu Y, Liang WH, Ou CQ, He JX, et al. Clinical Characteristics of Coronavirus Disease 2019 in China. The New England journal of medicine. 2020;382(18):1708-20.

2. Liu K, Zeng Y, Xie P, Ye X, Xu G, Liu J, et al. COVID-19 with cystic features on computed tomography: A case report. Medicine (Baltimore). 2020;99(18):e20175.

\section{Figure Legend}

Figure 1 A A, Coronal CT on admission showed bilateral patchy consolidation and GGO. B , Coronal CT on discharge showed a cystic feature in the left mediastinal side. Bilateral consolidation and GGO were attenuated. C , Coronal CT after four weeks from discharge showed additional cystic feature in the left epiphrenic side.D, Coronal CT after eight weeks from discharge showed disappearance of the cysts, bilateral consolidation, and GGO. 


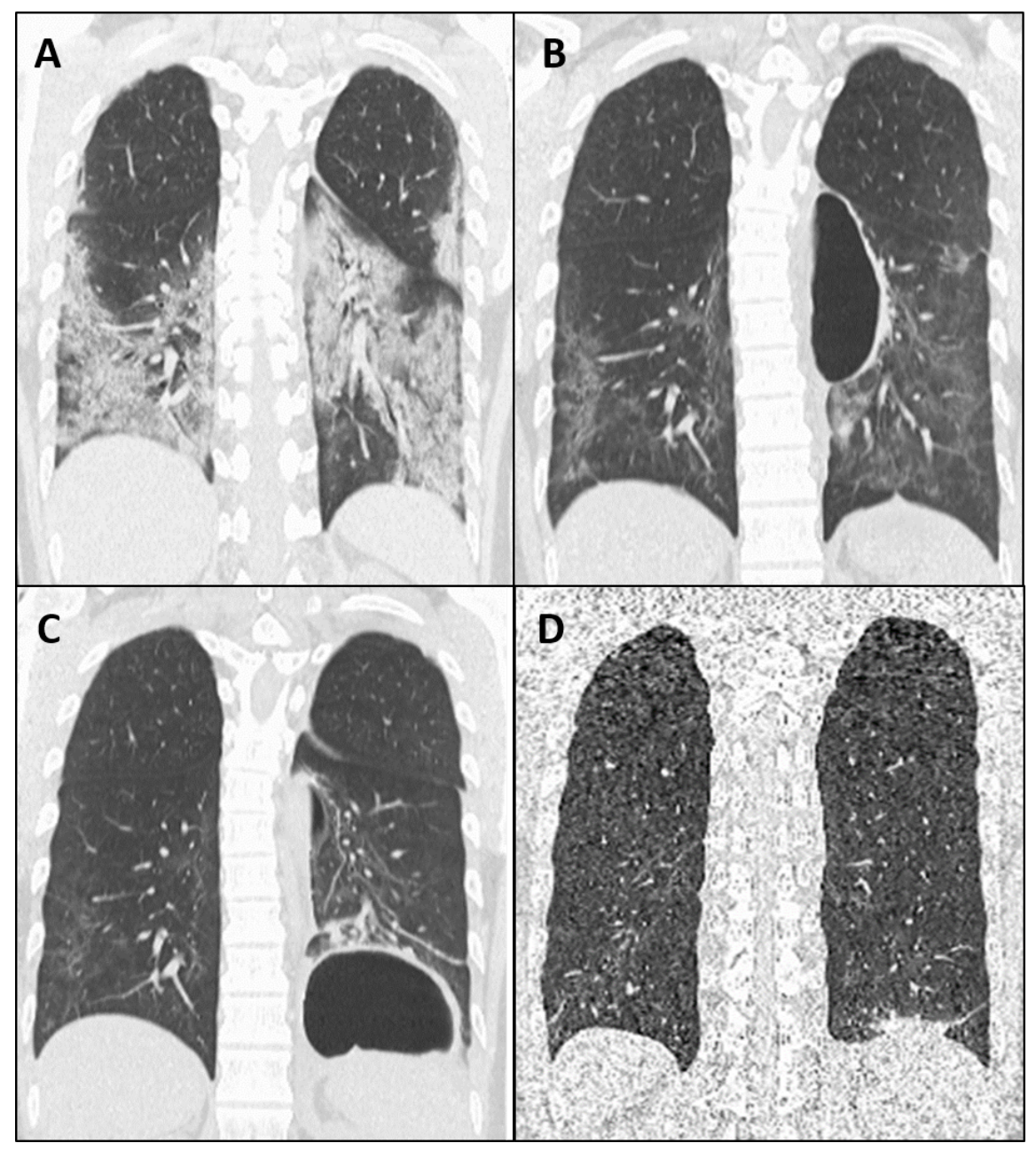

